\title{
WET CHEMICAL ETCHING MECHANISM OF SILICON
}

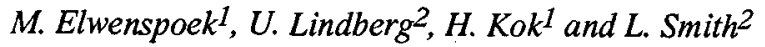 \\ 1MESA Research Institute, University of Twente. P.O. Box 217, NL-7500 AE Enschede, The Netherlands \\ $2_{2}$ Electronics Department, Institute of Technology, Uppsala University, Box 534, S-751 21 Sweden
}

\begin{abstract}
We review what can be said on wet chemical etching of single crystals from the viewpoint of the science of crystal growth. Starting point is that there are smooth and rough crystal surfaces. The kinetics of smooth faces is controlled by a nucleation barrier that is absent on rough faces. The latter therefore etch faster by orders of magnitude. The analysis of the diamond crystal structure reveals that the $\{111\}$ face is the only smooth face in this lattice - other faces might be smooth only because of surface reconstruction. In this way we explain the minimum of the etchrate in $\mathrm{KOH}: \mathrm{H}_{2} \mathrm{O}$ in the $<001>$ direction. Two critical predictions conceming the shape of the minimum of the etchrate close to $<001>$ and the transition from isotropic to anisotropic etching in $\mathrm{HF}: \mathrm{HNO}_{3}$ based solutions are tested experimentally. The results are in agreement with the theory.
\end{abstract}

\section{INTRODUCTION}

Anisotropic wet chemical etching of single crystalline silicon, gallium arsenide and quartz is one of the key technologies for the fabrication of microsystems. Yet the strong anisotropy of the etchrate in particular etching solutions (e.g. $\mathrm{KOH}: \mathrm{H}_{2} \mathrm{O}, \mathrm{EDP}, \mathrm{TMAH}$ ), and the isotropy in others (e.g. $\mathrm{HF}: \mathrm{HNO}_{3}: \mathrm{H}_{2} \mathrm{O}$ ) is poorly understood. Mostly the anisotropy of the etchrate is related to chemical reactions on the crystal surface oriented in different crystallographic directions. In this respect maybe the most advanced picture has been proposed by Seidel et al. [1]. They assumed that the complex formed by the attachment of an $\mathrm{OH}$-ion to the dangling bond, after the electron has been delivered to the solid state, changes the back-bond energy of the silicon atom with three back-bonds in a different way than in the situation when one has two $\mathrm{OH}$ attached to the silicon atom with two back-bonds. The difficult point however is that the silicon atoms have three backbonds also in the flat $\{110\}$ face, not only in the flat $\{111\}$ face; therefore, etchrate and activation energy in these crystallographic directions should be comparable in contrast to experimental evidence.
Recently it was suggested to use theories of crystal growth to analyse wet chemical etch data of single crystals [2]. Then a number of experimental results can be understood quite easily. The following points follow directly from standard theory of crystal growth:

-the isotropy of the etch rate in certain solutions and the anisotropy in others; criteria can be given that determine whether the etchrate is isotropic or anisotropic, and the criteria can be compared to experimental results

-the crystallographic orientations with the minimum etch rate (and growth rate)

-the amount of the anisotropy of the etchrate

-the anisotropy of the temperature dependence of the etchrate (the activation energy)

-the relation of the equilibrium form of the crystals (determined by the surface free energy = surface tension) to the anisotropy of the etchrate

-the shape of the minima of the etchrate, i.e. the variation of the etchrate as a function of the misorientation close to a minimum

In this paper we add a number of new experimental results which support the view given here. In particular, we have looked with greater detail at the dependency of the etchrate of silicon etched in $\mathrm{KOH}$ on the crystallographic orientation close to the $<100\rangle$ direction, and we studied the transition to anisotropic silicon etching in $\mathrm{HF}: \mathrm{HNO}_{3}: \mathrm{CH}_{3} \mathrm{COOH}$.

In the next section we briefly review the crystal growth point of view of wet chemical etching. We then describe our experiments and their results. A section of discussion and conclusions follows.

\section{THEORY}

In kinetics of crystal growth active sites for growth and dissolution play a key role. These active sites are atoms with as many bonds to the crystal as to the liquid (or gaseous) environment. Such a site is called a kink site. An atom in a kink site in a simple cubic lattice is shown in fig. 1a. The heavy shaded atom has three bonds to the crystal and three bonds to the liquid. In a dissolution 
situation it is commonly believed that this atom will diffuse over the surface (fig. 1b), until it either finds a kink position again or it desorps and diffuses away from the crystal in the liquid phase (indicated in fig. 1c). In a growth situation, an atom diffuses from the liquid to the crystal (fig. 1c), it diffuses over the crystal surface (fig. 1.b) until it is either desorped or it finds a kink site (fig. 1a).
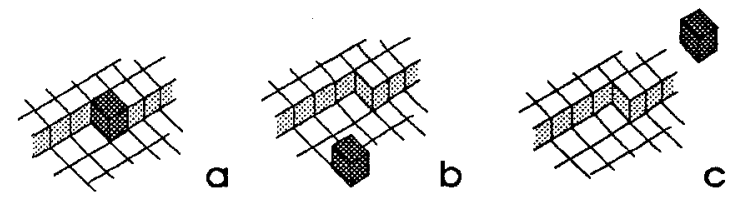

Fig. 1 Illustration of kink sites and the elementary kinetic processes for etching

(from a to c) and for growing (from $c$ to a) crystals

Kinetic rates (for growth and dissolution) thus depend critically on the number of kink sites on a crystal surface. This aspect was neglected so far in the discussion of etch rates of single crystals such as silicon, quartz and GaAs. Only parts of the total process have been considered: the chemical reaction rate (which is important for the adsorption process and the kink integration process, erroneously considered anisotropic), diffusion in the liquid solution (isotropic), and the thickness of the boundary layer. In our view, the most prominent anisotropy effect is due to the number of kink sites.

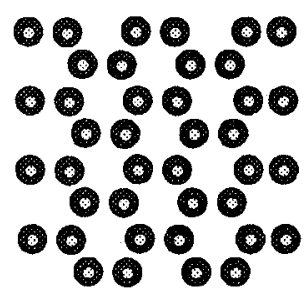

a

Fig. 2 Energy required to create a cavity-adatom pair on an unreconstructed \{111\} face of the diamond lattice. $\Delta E=2 \phi$

It is easily seen that the number of kink sites varies with the crystallographic orientation in a very dramatic way. The perfectly flat $\{111\}$ face in the diamond lattice has no kink positions (three backbonds, one dangling bond per atom), while on the $\{001\}$ face of silicon every atom has two back bonds and two dangling bonds - every position is a kink position.

Consider the energy difference of the two situations depicted in fig. 2. In fig. 2.a we show the $\{111\}$ silicon surface as one obtains by simply cutting the crystal along the $\{111\}$ plane. The dangling bonds are indicated by light dots. In fig. 2.b we have cut one atom out of the surface and placed it back somewhere else on the crystal surface. To do this one has to cut three bonds of strength $\phi$ each, but one bond is delivered by placing back the atom onto the surface. The energy difference $\Delta E=2 \phi$.
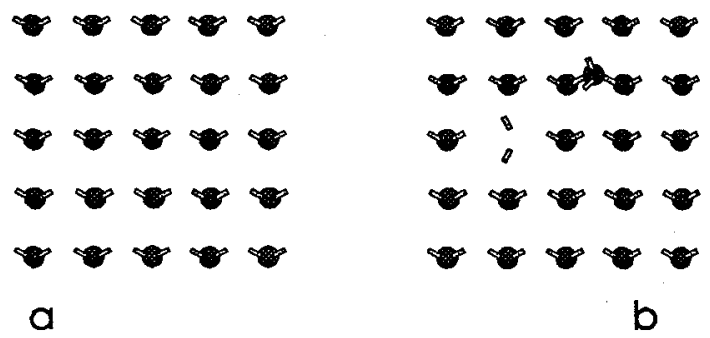

Fig. 3 Energy required to create a cavity-adatom pair on an unreconstructed \{001\} face in the diamond lattice. $\Delta E=0$

This is very much diffeent from the $\{001\}$ silicon face as can be seen in fig. 3. The same operation - creating an adatom-cavity pair - now costs no energy, because one has to break two bonds in order to remove an atom from the $\{001\}$ face, but one gets them back by placing it back to any position on this face.

The energy difference $\Delta \mathrm{E}$ divided by $\mathrm{kT}$ (absolute temperature times Boltzmann constant) is known as the $\alpha$-factor of Jackson (see, e.g. [3]) and plays a key role in theory of crystal growth. At sufficiently low temperature $\mathrm{kT} \alpha$ is proportional to the step free energy $\gamma$. The essential difference between the $\{111\}$ and the $\{001\}$ silicon face is: In equilibrium the $\{111\}$ face is smooth at sufficiently small temperature and the $\{001\}$ is rough. The step free energy $\gamma$ is finite of the $\{111\}$, and zero of the $\{001\}$ face of silicon. The number of cavity - adatom pairs is proportional to $\exp -\alpha$. This number is very small on the $\{111\}$ silicon face at low temperature, but 1 on the $\{001\}$ silicon face at any temperature.

The $\{110\}$ face of silicon is a so-called stepped face, c.f. e.g. [4]. The surface consists of chains in the $\{110\}$ direction which are not laterally connected. It is possible to take a chain and replace it somewhere else on the surface without doing work. Consequently, the $\{110\}$ is rough [4].

There is a phase transition at a temperature $T_{R}$ between a smooth and a rough crystal face, above which $\gamma$ vanishes (for a review see [5]). For simple systems this is a continuous phase transition. $\alpha_{\mathrm{R}}=\Delta \mathrm{E} / \mathrm{kT} \mathrm{T}_{\mathrm{R}}$ only depends on the symmetry of crystal faces; in the diamond lattice only the $\{111\}$ face has a finite $\alpha_{R}(\sim 4)$ and $\alpha_{R}$ of all other faces (thus including $\{100\}$ and $\{110\}$ ) are infinite $[6,7]$, provided that there is no surface reconstruction. Surface reconstruction however is important and will be addressed below.

A crystal dissolves or grows only in a non-equilibrium situation. There must be a difference in chemical potential $\Delta \mu$ of the atoms in the crystalline phase and in the liquid phase. Etch- and growth rates depend on $\Delta \mu$, and the 
type of dependency differs greatly for smooth and for rough faces $[4,5]$.

In order to remove atoms from a smooth face, one has to create a step. Consider a cavity that contains $\mathrm{N}$ vacancies ( $\mathrm{N}$ atoms have been removed). This implies a gain of the free energy of the system which is equal to $N \Delta \mu$. Obviously, $\mathrm{N}$ is proportional to the surface of the cavity, and for a cavity of circular shape therefore proportional to the square of the radius. But a step has been created, and a positive step free energy arises, that is proportional to the length of the step. Hence this term is linearly proportional to the radius of the cavity. Adding these contributions, we get a dependency of the total free energy change by creating a cavity that has a maximum at a critical radius. There is a nucleation barrier, similar to e.g. condensation of water droplets from supersaturated steam. Only cavities larger than the critical size have a good chance to grow; too small cavities most probably will disappear. More details are given in [2].

In order to etch therefore one has to overcome a twodimensional nucleation barrier. Etch rates will contain a factor $\exp \{-\Delta G * / k T\}$, where $\Delta G *$ is the free energy of a critical nucleus,

$$
\Delta \mathrm{G}^{*}=\xi \gamma^{2} / \Delta \mu,
$$

with $\gamma$ the step free energy and $\xi$ a geometrical factor roughly of the order of the surface an atom occupies in the crystal face. If $\Delta G^{*} / \mathrm{kT}_{\mathrm{T}}$ is large the etchrate will be very small. This is the case, both for small undersaturation and for large step free energies.

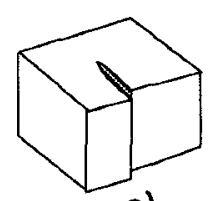

a)

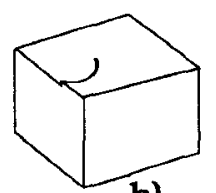

b)

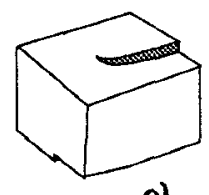

c)
Fig. 4. A screw dislocation gives rise to a step on the crystal surface (a). The step moves when the crystal grows (b) and if it is etched (c)

There is a second mechanism for creating steps, thereby creating kink positions, which makes etching of smooth faces possible [8]. In fig. 4 a we depict a screw dislocation. Any screw dislocation that crosses a surface gives rise to a step. This step moves if the crystal grows (b) or if it is etched (c).

When looking to a crystal surface with a phase contrast or interference contrast microscope, usually one sees the resulting growth spirals. They can also be seen on crystals during etching, see e.g. $[9,10]$. The growth/etch rate of imperfect crystals is proportional to $\Delta \mu^{2}$ [8].

A rough crystal face etches or grows with a rate directly proportional to $\Delta \mu$ (e.g. [5]).

This theory has the following consequences: (i) Rough crystal faces etch much faster than smooth crystal faces. Elementary analysis predicts that the only smooth face of the diamond lattice is $\{111\}$ [6]. There may be more flat faces due to surface reconstruction and/or absorption [10], prominant candidates being the $\{001\}$ and $\{110\}$. The $\{111\}$ remains the face with the largest step free energy and should etch with the slowest rate.

(ii) The activation energy of smooth crystal faces contains the free energy of a critical nucleus. The energy barrier of the chemical reaction and transport in the liquid contribute to the activation energy. The latter contributions are isotropic, and the former is anisotropic, and absent on rough faces. The larger the step free energy, the larger the activation energy, the smaller the etchrate. The $\{111\}$ faces should have the largest activation energy. This is in perfect agreement with experimental results.

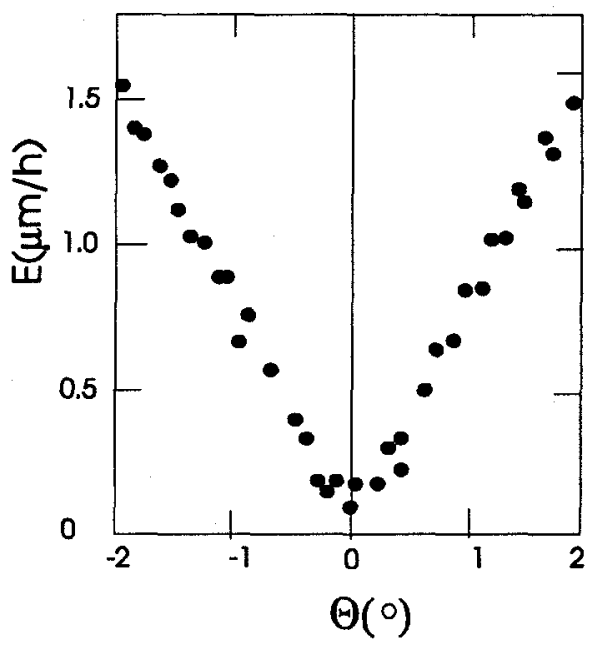

Fig. 5. Underetch rate variation in $<001>$ oriented silicon wafers close to [111\}, redrawn from fig. 11 of ref. [1]. EDP type, $69^{\circ} \mathrm{C}$.

(iii) $\Delta \mu$ and $\gamma$ both depend on the type of etchant. These parameters might provide the clue to understand the variation of the etchrate, degree of anisotropy, temperature dependence with the etch solution. Here further research is required.

(iv) A misalignment close to smooth faces implies steps; there is no need of nucleation in order to etch. Since the density of steps is proportional to the angle of misalignment, the etchrate should be proportional to the angle, provided the distance between steps is not too large, so that nucleation of new cavities is very probable. This has been observed for the etchrate close to $\{111\}[1]$, and we reproduce Seidel's results in fig. 5 .

(v) Analysis of etch data can provide clues on the physical state of real crystal surfaces. Actually the \{111\} faces of silicon may not look like the honeycomb structure shown in fig. 2, but there is a -meanwhile famous - 7 by 7 surface reconstruction [11]. Also there 
is evidence that the $\{001\}$ faces are reconstructed [10]. The idea is that the dangling bonds feel each other, and these additional bonds make the step free energy of the $\{001\}$ face finite. However, direct evidence for the surface reconstruction is only available for silicon surfaces in contact with vacuum - in contact with water or $\mathrm{KOH}$ or whatever, it could be dramatically different.

(vi) The equilibrium form of a crystal is dominated by the flat faces; which are the faces having the smallest surface free energy ("surface tension") [12]. These faces have large step free energies, they grow and etch slowly. Therefore, once the surface tension or the equilibrium form is known, one has a good guess of the growth form and of the faces that etch slowly.

(vii) If the undersaturation becomes so large that $\Delta \mathrm{G}^{*} \ll$ $\mathrm{kT}$, the nucleation barrier breaks down. Each singleatom-cavity acts as a nucleus, which are made by thermal fluctuation in vast numbers. The face in question etches with a rate comparable to the etchrate of a rough face. This situation is known as kinetic roughening [13]. If all faces are kinetically rough, the etch rate becomes isotropic. We expect that this is the case in $\mathrm{HF}: \mathrm{HNO}_{3}$ based etchants. A change of the degree of anisotropy by a change of $\Delta \mu$ can be expected in two cases: In the very beginning of e.g. $\mathrm{KOH}$-etching the undersaturation is very large, the etching should be isotropic. The time however might be too short in order to observe this effect. But etching in $\mathrm{HF}: \mathrm{HNO}_{3}$ based etchants should show some anisotropy if one etches sufficiently long, so that the undersaturation decreases below the level of kinetic roughening.

In this paper we study two effects which are expected from the theory outlined above:

-We investigated the shape of the minimum close to the $\{001\}$ direction. If the $\{001\}$ silicon face is smooth, the etchrate should vary linearly with the angle of misorientation if the minimum is caused by a nucleation barrier. The minimum should be like a cusp except very close to the $\{001\}$ orientation.

-We investigated the nature of etching in an isotropic etching solution, monitoring the degree of anisotropy as a function of etching time.

\section{EXPERIMENTS}

\section{III.1. Anisotropic etching}

From (iv) of section II it follows that the etchrate $\mathrm{E}$ and growth rate $\mathbf{G}$ vary proportional to the absolute value of the angle of misorientation $\Delta \Theta$,

$$
E \sim G \sim|\Delta \theta|
$$

In agreement with experimental results, see fig.5 [1]. Similar results have been published by Kendall [14]. However, the situation close to the $\langle 100\rangle$ directions is unclear. In the $\langle 100\rangle$ - direction there is also a minimum if one etches silicon in $\mathrm{KOH}$ and $\mathrm{NaOH}$ [15]. In situations where the minimum is steep enough it is possible to micromachine vertical $<100\rangle$ walls in $<001\rangle$ oriented wafers [16]. Mirror like flat $\{100\}$-faces have been reported $[16,17]$ at particular etching conditions $(35$ wt $\% \mathrm{KOH}, 80^{\circ} \mathrm{C}$ ). Possibly the $\{100\}$ are flat under these conditions in which case eq. (1) would apply.

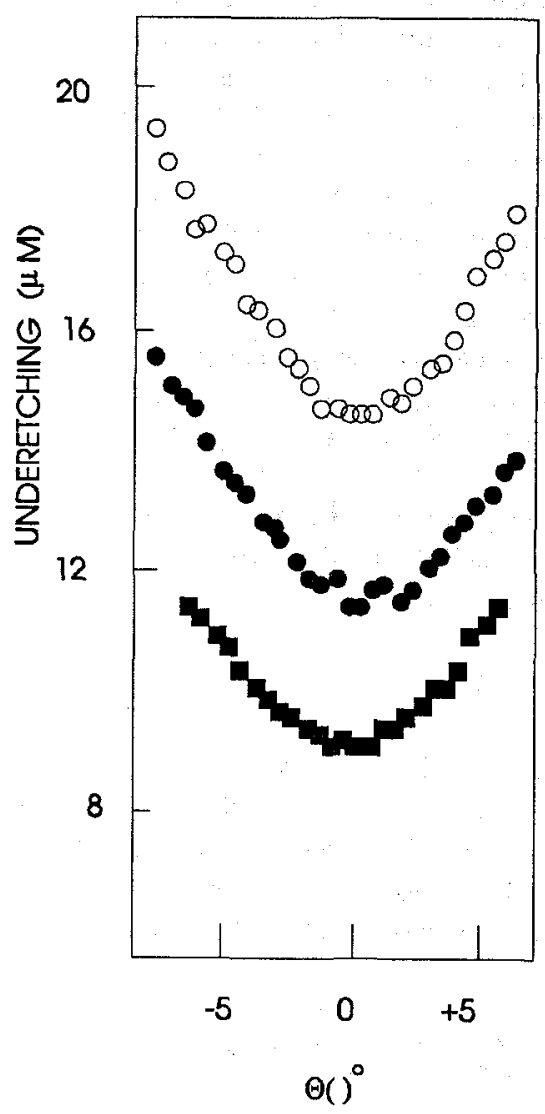

Fig.6. Underetching close to $<100>$ on a $<001>$ oriented silicon wafer in aqueous $\mathrm{KOH}(40 \mathrm{gr} / 100 \mathrm{ml})$ open points: $30^{\circ} \mathrm{C}, 300 \mathrm{~min}$; full squares: $70^{\circ} \mathrm{C}, 15 \mathrm{~min}$; full points: $108^{\circ} \mathrm{C}, 3 \mathrm{~min}$

We conducted experiments to test this question. A mask was designed that consisted of rectangular openings (250 $x 50 \mu \mathrm{m}$ ), the long side of which made an angle with the $<100\rangle$ direction on an $<001\rangle$ oriented wafer. The angular resolution was $0.5^{\circ}$. Etching in $\mathrm{KOH}$ results in grooves bounded by $\{001\}$ planes. The experiments were performed in aqueous $\mathrm{KOH}(40 \mathrm{gr} / 100 \mathrm{ml})$. Temperature and etch times are given in table 1.

\begin{tabular}{|cc|}
\hline $\mathrm{T}\left({ }^{\circ} \mathrm{C}\right)$ & $\mathrm{t}(\mathrm{min})$ \\
30 & 300 \\
70 & 15 \\
108 & 3 \\
\hline
\end{tabular}

The wafers were placed vertically in the etch vessel. 
The results of the etch rates are shown in fig. 6 . It is seen that except at a small region around the $<001\rangle$ of a width of $2-3^{\circ}$, the underetch rates vary linearly with the angle for all temperatures. The underetch rates are not symmetrical with respect to the minimum, a trend that is more pronounced at higher temperature. This is probably due to convection in the etchant. Being placed vertically, the flow of hydrogen bubbles may cause convection that is different on either side of the grooves.

It may be interesting to note the following: When similar experiments are performed on $\langle 110\rangle$ wafers (etching vertical grooves bounded by $\{111\}$ planes), one finds that the slightly misoriented $\{111\}$ planes are composed of terraces and macro steps [14]. If macrosteps were present on the $\{100\}$ planes in our experiments they are too shallow to be detected by the optical microscope used for investigations.

\section{III.2. Isotropic etching}

The etchrate of smooth faces (e.g. in the $<111\rangle$ direction) is determined by a nucleation barrier, the height of which is given by eq.(1). This barrier can be overcome if $\Delta \mu$ is large enough $\left(\mathrm{G}^{*}<\mathrm{kT}\right)$. The phenomena associated with this condition are well known and are referred to as kinetic roughening [13]. Isotropic etching of single crystals obviously requires conditions of kinetic roughening, because then the etchrate is no more dominated by a nucleation barrier but by transport processes in the solution and the chemical reaction rate. If this was true there should be a transition from isotropic to anisotropic etching if the undersaturation $\Delta \mu$ becomes too small. This must be the case if one etches long enough. We think that this experiment is critical for the mechanism of anisotropic etching proposed here.

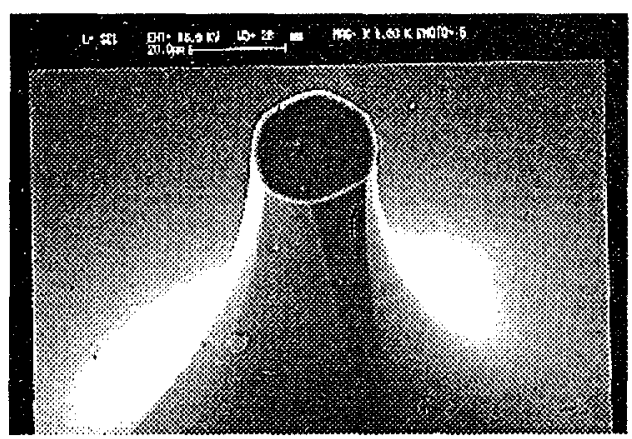

Fig.6 SEM photograph of a column etched under a circular mask in a $\langle 111\rangle$ oriented wafer after etching $100 \mu \mathrm{m}$ deep in the silicon. In earlier stages the column stays cylindrical as one expects for isotropic etching. Etching time was 40 minutes.

In fig. 6 we show this effect. Circular oxide $+\mathrm{CrAu}$ masks on a $2^{\prime \prime}<111>$ oriented silicon wafer were underetched in $\mathrm{HF}: \mathrm{HNO}_{3}: \mathrm{CH}_{3} \mathrm{COOH}(2: 15: 5)$ at $25^{\circ} \mathrm{C}$ in a stirred solution. The volume of the solution was 400 $\mathrm{ml}$. The wafers were placed horizontally in the beaker. While in the beginning the underetched columns are perfectly cylindrical, after 20 minutes, having etched 100 $\mu \mathrm{m}$ deep into the $<111>$ oriented wafer, flat regions start to develop showing the six fold symmetry one expects for crystallographic effects on the $\{111\}$ silicon face. The vertices seen in fig. 6 are oriented to the $\langle 111\rangle$ directions demonstrating that the etchrate has a minimum in the this direction.

Similarly, it has been observed by Hashimoto et al. [18] that isotropic etching etchants may etch anisotropically under certain circumstances. They etched silicon through small holes in the mask, trying to etch semi-spherical cavities. If the hole in the mask was large enough they succeeded but they observed that the cavities revealed some anisotropy if the holes in the mask were very small. This is a case where ageing of the solution may be visible, since the small hole in the mask hinders the transport of fresh solution to the silicon surface. These results give indirect evidence that when decreasing undersaturation the etchrate becomes anisotropic.

Here we have now direct evidence that kinetic roughening is responsible for the isotropic character of etching silicon in $\mathrm{HF}: \mathrm{HNO}_{3}$ based etchants.

\section{DISCUSSION}

The minimum of the etch rate at the $<100\rangle$ direction implies in our view that the $\{100\}$ faces are flat when they are in contact with $\mathrm{KOH}$ solutions investigated in our experiments. This is only possible if the surfaces are either reconstructed or if there is an adsorption layer on the $\{100\}$ faces that stabilise in some way the surface [10]. To our best knowledge, this is the first time that some evidence of surface reconstruction of silicon surfaces in contact with an etching solution is given.

Certainly, the step free energy on $\{100\}$ is considerably smaller than on the $\{111\}$ faces, as evidenced by the large etch rate of the $\{100\}$. This is consistent with the absence of high steps on slightly disoriented $\{100\}$ faces. These high steps are created by "collisions" of steps, a process that is strongly enhanced by adsorption of impurities at the surface.

The temperature dependence of the slope of the etchrate, $\mathrm{dE} / \mathrm{d} \Theta$, is dominated by velocity of steps; no nucleation barrier should be visible in the temperature dependence. On the other hand, the nucleation barrier should be visible at the small flat portion of the plots in fig. 6 , if nucleation plays the decisive role for the etching. However, the activation energies are both of the order of $0.53 \mathrm{eV}$ (Seidel et al. report $0.58 \mathrm{eV}$ [1]), hence either the nucleation barrier is very small or dissolution spirals dominate the etch mechanism in the $\langle 100\rangle$ direction.

The latter conclusion is supported by the temperature dependence of the width of the flat parts in fig. 6; it does not seem to depend on temperature at all. 
The flat portion arises from steps that are not created by misalignment, but spontaneously either by presence of spirals due to screw dislocations or by nucleation. At the transition from the flat portion to the linear portion the density of steps due to both mechanisms is comparable. It can be shown that the width of the flat portion $\Delta \theta$ is proportional to

$\Delta \theta \sim \exp -\Delta G * / k T$

if nucleation was dominant for the etchrate. In the case that screw dislocations were dominant, the wavelength of the steptrain due to misorientation must equal the wavelength of the steptrain of the spiral. The latter is given by $19 r^{*}$ [8], where $r^{*}$ is the radius of the critical nucleus, $\mathrm{r}^{*} \sim \gamma / \Delta \mu$, and we find that

$\Delta \Theta \sim \Delta \mu / \gamma$

Roughly, $\Delta \mu \sim \mathrm{kT}$, in agreement with the small temperature dependence of $\Delta \boldsymbol{\theta}$. We arrive at the conclusion, that - if our model of the etch mechanism is correct - spirals dominate the etching of $\{001\}$-faces.

We consider the transition of isotropic etching to anisotropic etching when the undersaturation decreases as a critical test of the crystal growth view on wet chemical etching. The agreement of experimental results with the theoretical prediction seems to be quite convincing. However, in future more experiments have to be conducted to give more quantitative insight. At this moment our knowledge of especially thermodynamic data is to poor to allow a quantitative discussion. If these data were known, it would be possible to estimate the edge free energy of steps on the $\{111\}$ faces of silicon in the isotropic etching etchants from the observation of the transition from isotropic etching to anisotropic etching. From eq. (2) we have, from the requirement $G^{*} \cong \Delta \mu$,

$\gamma \cong\{\mathrm{kT} \Delta \mu\}^{1 / 2}$

For ideal solutions, and not too far from equilibrium, we have $\Delta \mu \sim \mathrm{kT}\left(\mathrm{X}_{\mathrm{eq}}-\mathrm{X}\right) / \mathrm{X} . \mathrm{X}$ refers to the actual molefraction and $X_{e q}$ to the equilibrium mole fraction of the etchproducts. From our experiments we can estimate the actual $X$ where the transition to anisotropic etching occurs, but we do not know $X_{\text {eq }} \cdot \gamma$ in turn should be related to the heat of dissolution, which also seems to be not known.

\section{CONCLUSIONS}

We think that the crystal growth view of isotropic and anisotropic wet chemical etching of single crystals provides an important step towards the understanding of the physical-chemical mechanism of etch processes. The main features of the process as listed in the introduction can be understood at least qualitatively. For a more quantitative discussion more thermodynamic properties of the etch solution must be known. The experimental results of isotropic and anisotropic etching support the model presented in this paper.

\section{REFERENCES}

[1] H. Seidel, L. Csepregi, A. Heuberger and H. Baumgärtel, J. Electrochem Soc. 137, 3612 (1990)

[2] M. Elwenspoek, J. Electrochem Soc. 140, 2075 (1993)

[3] K.A. Jackson, in: Crystal Growth, ed. H.S. Peiser, Pergamon Press, Oxford (1967), p17

[4] e.g. P. Hartman (ed.) Crystal growth, An Introduction, North Holland, Amsterdam (1973)

[5] P. Bennema, J. Crystal Growth 69, 182 (1984)

[6] P. Hartman, Z. Kristallogr. 121, 78 (1965)

[7] J.P. van der Eerden, Phys. Rev. B, 13, 4942 (1976)

[8] W.K. Burton, N. Cabrera and F.C. Frank, Phil. Trans. Roy. Soc. London A243, 299 (1951)

[9] e.g. I. Sunagawa and P. Bennema, J. Crystal Growth 53, 490 (1981)

[10] LJ. Giling and W.J.P. van Enckevort, Surf. Sci. 161, 567 (1985)

[11] R. Wiesendanger, G. Tarrach, D. Bürgler H.-J. Güntherrodt, Europhys. Lett. 12, 57 (1990)

[12] G. Wulff, Z. Krist. 34, 449 (1901)

[13] M. Elwenspoek and J.P. van der Eerden, J. Phys. A 20, 669 (1987)

[14] D.L. Kendall, J. Appl. Phys. 26, 195 (1975)

[15] J. Smits, J. Micro Electromechanical Systems 2, 82 (1993)

[16] L. Ternez, Y. Bäcklund, J. Tirén and O'Connnell, Sensors and Materials 1-6, 313 (1989)

[17] L. Rosengren, L. Smith and Y. Bäcklund, Sensors and Actuators (in the press)

[18] H. Hashimoto, S. Tanaka and K. Sato, Proc. Transducers '91, San Francisco, Ca., U.S.A., June 23 - 27, (1991), pp. 853 